\title{
Heterodoped Nanoparticles as Dual-Mode Contrast Agent for MRI
}

\author{
Akbar Alipour ${ }^{1,2,3}$, Vijay Kumar Sharma ${ }^{2,6}$, Zeliha Soran-Erdem², Yusuf Kelestemur ${ }^{2}$, Zaliha Gamze \\ Aykut ${ }^{5}$, and Hilmi Volkan Demir ${ }^{1,2,4,6}$ \\ ${ }^{I}$ Department of Electrical and Electronics Engineering, ${ }^{2} U N A M$ - Institute of Materials Science and \\ Nanotechnology, ${ }^{3}$ UMRAM - National Magnetic Resonance Research Center, ${ }^{4}$ Department of Physics, \\ ${ }^{5}$ Department of Molecular Biology and Genetics, Animal Research Laboratory, Bilkent University, 06800 \\ ANKARA, Turkey, ${ }^{6}$ School of Mathematical and Physical Sciences, Nanyang Technological University, \\ Singapore 639798, Singapore.
}

\begin{abstract}
The purpose of this work is to synthesize $\mathrm{Mn}-\mathrm{Fe}$ heterodoped $\mathrm{ZnSe}$ tetrapod nanocrystals (NCs) as dualmode MRI contrast agent to offer synergetic beneficial over the single contrast tracer. Also, in vivo feasibility of the Mn-Fe heterodoped ZnSe tetrapod NCs as a dualmode contrast agent has been studied.

Keywords: co-doped nanocrystals, superparamagnetic, paramagnetic, magnetic resonance imaging, contrast agents

\section{Introduction}

MRI contrast agents are generally in the form of positive (T1) and negative (T2) contrast agents. Positive contrast agents with high signal intensity enhance the resolution between the tissues. Negative contrast agents typically are used for lesion detection. T1-T2 dual-modal strategy can result in complementary diagnosis information by the advantages of positive and negative contrast effect. In this study, we report heterodoped nanoparticles that simultaneously enhance contrast in both T1- and T2-weighted MRI images. The in vivo T1- and T2-weighted MRI images promise the great potential of these nanoparticles as dual-mode MRI contrast agent in clinical applications.
\end{abstract}

\section{Method}

Superior soft tissue contrast is a major advantage of the magnetic resonance imaging (MRI). However, even MRI fails to provide adequate discrimination between tissue structures. In these circumstances, T1 and T2 relaxation enhancing contrast agents can be employed. T1 contrast agents generally increase MR signal intensity consequently lead to high contrast-to-noise ratio [1]. T2 contrast agents are generally used for lesion detection [2]. T1-T2 dual-modal imaging can result in complementary diagnosis information by the advantages of positive and negative contrast effect [3]. In this work, we reported new nanoparticles that simultaneously enhance contrast in both $\mathrm{T} 1$ - and T2weighted MRI images.

\section{Results}

Mn-Fe heterodopted ZnSe tetrapod NCs were fabricated using nucleation doping method. In our synthesis, Mn is generally placed at the center of the ZnSe NCs while Fe stands on the branches (Figure 1a). The crystal structure and morphology of these NCs were investigated using transmission electron microscopy (TEM). The TEM image of the NCs is represented in Figure 1b. TheSE nanotetrapods with core diameter of $\sim 4.1 \mathrm{~nm}$, thickness of $\sim 2.5 \mathrm{~nm}$, and a branch length of $\sim 5.8 \mathrm{~nm}$, have an average size of $\sim 16 \mathrm{~nm}$. The magnetic analysis (M-H curve) showed that these NCs represent paramagnetic behavior (Figure 2a), while Fe co-doping induced super paramagnetic behavior in addition to the intrinsic paramagnetic behavior of these NCs (Figure 2b). 
Therefore, the simultaneous magnetic (paramagnetic and super paramagnetic) phases of the $\mathrm{Mn}-\mathrm{Fe}$ heterodopted $\mathrm{ZnSe}$ tetrapod NCs increases the possible dual-mode contrast enhancement effect of these NCs in
T1-and T2-weighted MRI imaging. T1 and T2 relaxation times of the water-diluted samples were obtained using inversion recovery sequences and multiecho spin-echo sequences, respectively on 3T Siemens MRI scanner. Figure $3 \mathrm{a}$ and, $\mathrm{b}$ show the T1-

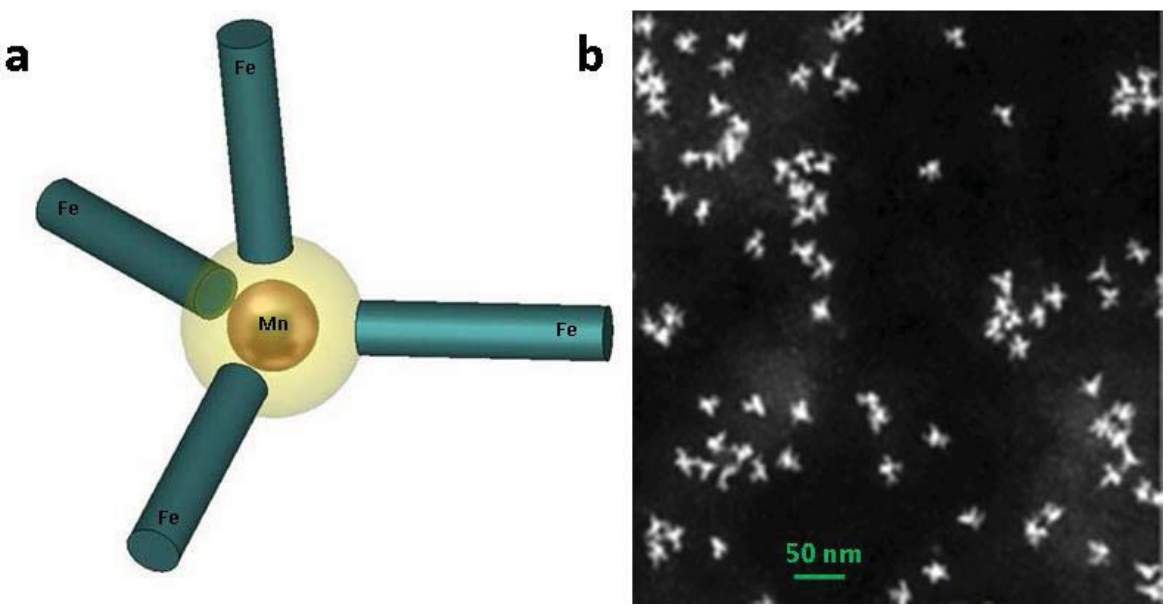

Fig1. Schematic of the synthesized Mn:Fe heterodoped ZnSe nanotetrapods (a). TEM image of the nanotetrapods (b).
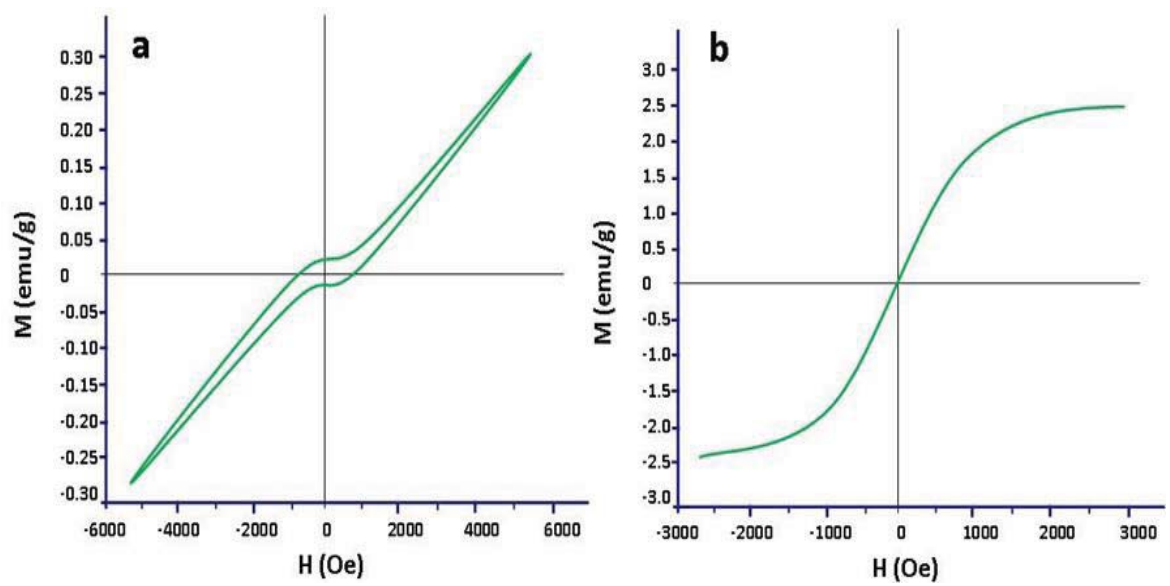

Fig 2. M-H curve of the Mn-Fe heterodopted $\mathrm{ZnSe} \mathrm{NCs}$ without Fe co-doping (a), with Fe co-doping (b).

and T2-weighted in vitro images of DI water and the systematically diluted samples. The gradually increasing signal intensity in T1-weighted images with the increasing Mn concentration represents the positive contrast enhancement. On the other hand, we observed that the signal intensity gradually decreases with the increasing Fe concentration in T2-weighted images. The longitudinal (r1) and transvers (r2) relaxivity rates were calculated from the slop of $(1 / \mathrm{T} 1,2-1 / \mathrm{T} 0)$ vs $\mathrm{Mn} / \mathrm{Fe}$ concentration (Figure 3c,d). Therefore, the in vitro MRI 
images and relaxivity analysis results demonstrate the simultaneous contrast enhancement of our synthesized

\section{a}
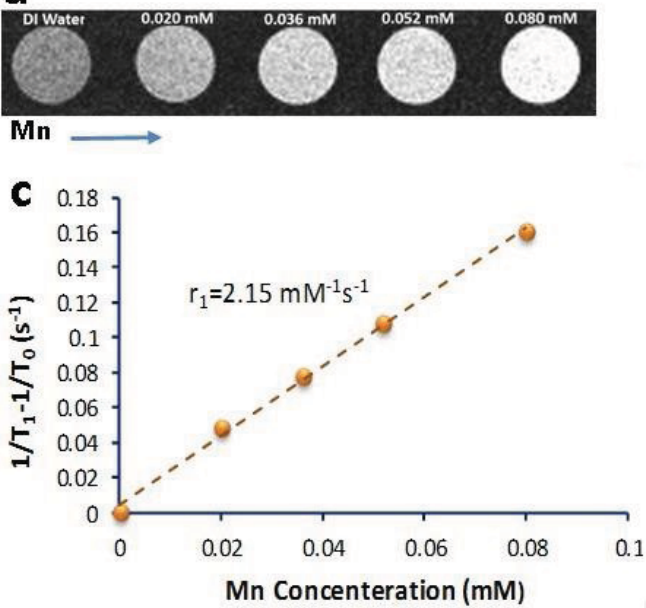

NCs.
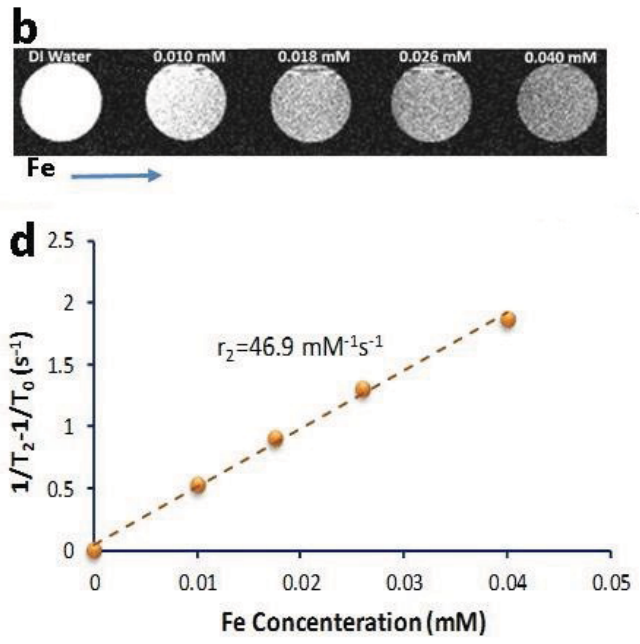

Fig 3. $\mathrm{T}_{1}$-weighted (a) and $\mathrm{T}_{2}$-weighted (b) in vitro MRI images of the NCs at different $\mathrm{Mn}$ and Fe concentration, respectively. $\mathrm{r}_{1}$ and $\mathrm{r}_{2}$ relaxivity analysis vs, $\mathrm{Mn}$ and $\mathrm{Fe}$ concentration at room temperature (c,d).

The in vitro cytotoxicity analysis was carried on using L929 cell line and results showed that these NCs are not cytotoxic up to $100 \mu \mathrm{g} / \mathrm{mL}$ concentration.
In vivo experiments were conducted on a SparagueDawley rat using 3T MRI scanner. T1-weighted spinecho sequences $(\mathrm{TR} / \mathrm{TE}=550 / 11 \mathrm{~ms})$ and $\mathrm{T} 2$-weighted spin-echo sequences $(\mathrm{TR} / \mathrm{TE}=4420 / 92 \mathrm{~ms})$ were used to obtain MRI images. Imaging was performed before and after IV injection of the NCs with the dose of $1 \mathrm{mg} \mathrm{kg}-1$.

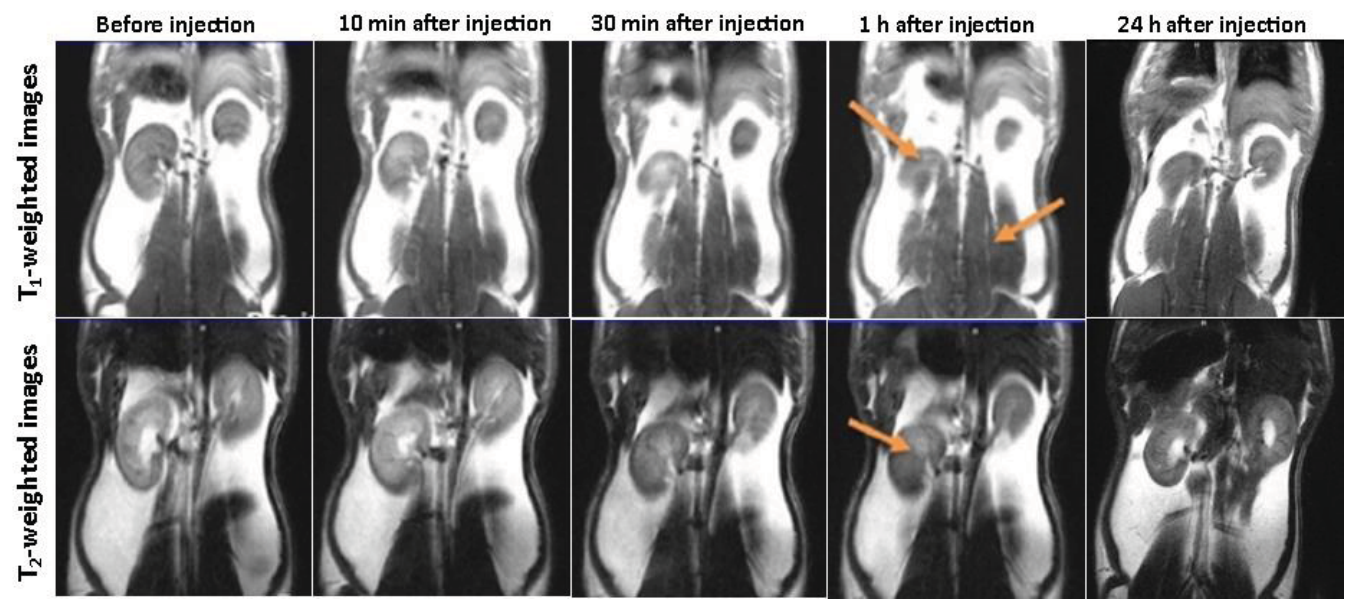

Fig 4. T1- and T2-weighted MRI images of the rat abdomen. MRI images were obtained before and $10 \mathrm{~min}, 30 \mathrm{~min}, 1$ hour, and 24 hours after IV injection of the NCs, respectively. Arrows show the interested regions. 
The coronal images of the kidney and veins were obtained before and $10 \mathrm{~min}, 30 \mathrm{~min}$, and 1 hour after injection, respectively. MR imaging results show progressively brighter and darker traces on $\mathrm{T} 1$ weighted and T2-weighted images, respectively (Figure 4). Which represent the in vivo feasibility of a single contrast tracer as simultaneous contrast enhancement in MRI. Comparison of the MRI images after 24 hour at the same position reveals that most of the injected nanocrystals were removed from the body.

In summary, we have shown that our synthesized Mn-Fe heterodoped ZnSe tetrapod NCs simultaneously can improve the contrast in both T1- and T2-weighted MRI images. Thus, these NCs as a single tracer with dualmode MRI contrast effect can be considered as a potential candidate to apply for clinical diagnosis.

\section{Kaynaklar}

[1] Pan D, Schmieder A. H, Wickline S. A, Lanza G. M et al. Manganese-Based MRI Contrast Agents: Past, Present, and Future. Tetrahedron. 2011;67 (44): $8431-8444$.

[2] Corot C, Warlin Det al. Superparamagnetic Iron Oxide Nanoparticles for MRI: Contrast Media Pharmaceutical Company R\&D Perspective. WIREs Nanomed. Nanobiotechnol. 2013; 5 (5): 411-422.

[3] Sharma V, Alipour A, Soran-Erdem Z, Kelestemur Y, Aykut G, Demir H, et al. Flurescent heterodoped nanotetrapods as synergistically enhancing positice and negative magnetic resonance imaging contrast agents. ACS applied materials and interfaces, 2016; 8:1235212359. 